\title{
Reconhecendo Grafos com até 1 Cruzamento
}

\author{
André Carvalho Silva ${ }^{1}$, Orlando Lee ${ }^{1 *}$ \\ ${ }^{1}$ Instituto de Computação - Universidade Estadual de Campinas (UNICAMP) \\ Campinas, São Paulo - Brasil \\ andre.silva, leedic.unicamp.br
}

\begin{abstract}
The crossing number $\mathrm{cr}(G)$ of a graph $G$ is the least number of crossings over all possible drawings of $G$. This paper describes a minor improvement over a naive algorithm for deciding if $\mathrm{cr}(G) \leq 1$.

Resumo. O número de cruzamentos de um grafo $G$ é o menor número de cruzamentos dentre todos os desenhos de G. Este artigo descreve uma pequena melhora para um algoritmo ingênuo para decidir se $\operatorname{cr}(G) \leq 1$.
\end{abstract}

\section{Introdução}

Um desenho $D$ de um grafo $G=(V(G), E(G))$ é a união das imagens de:

- uma função injetiva $\phi: V(G) \rightarrow \mathbb{R}^{2}$, e

- uma função $\phi_{e}$ para cada aresta $e=u v$, tal que $\phi_{e}$ é um mapeamento contínuo entre o intervalo real $[0,1]$ e um subconjunto do $\mathbb{R}^{2}, \phi_{e}(0)=\phi(u)$ e $\phi_{e}(1)=\phi(v)$, ou vice-versa, e $\phi(V) \cap \phi_{e}([0,1] \backslash\{0,1\})=\emptyset$.

No texto, não faremos distinção entre as arestas e vértices do grafo e o conjunto de pontos representando elas em $D$. Seja $H$ um subgrafo de $G$, usamos a notação $D[H]$ para representar o desenho de $H$ em $D$.

Seja $D$ um desenho de um grafo $G$, um cruzamento é uma interseção entre o interior de duas arestas. O número de cruzamentos $\operatorname{cr}(D)$ de $D$ é o número de interseções entre arestas em $D$. O número de cruzamentos $\operatorname{cr}(G)$ de um grafo $G$ é o menor $\operatorname{cr}(D)$ dentre todos os desenhos $D$ de $G$.

O número de cruzamentos de um grafo tem aplicações práticas nas áreas de VLSI (Very Large Scale Integration) e de desenhos de grafos (graph drawing). Leighton [Leighton 1983] mostrou que a área necessária para representar um circuito elétrico está intrinsecamente relacionada ao número de cruzamentos do grafo que representa o circuito. Purchase [Purchase 1997] fez uma pesquisa mostrando que o desenhos de grafos que minimizam o número de cruzamentos são os mais fáceis de entender visualmente.

Como apontado por Székely [Székely 1997], resultados sobre o número de cruzamentos de grafos podem ser utilizados para provar, de forma simples, alguns problemas difíceis de geometria discreta.

Dado um grafo $G$ e um inteiro $k$, determinar se $\operatorname{cr}(G) \leq k$ é um problema NPCompleto [Garey and Johnson 1983]. Sabe-se que o problema é fixed-parameter tractable para $k$ fixo [Grohe 2001]. Informalmente, isto significa que para cada $k$ fixo existe um algoritmo polinomial que decide $\operatorname{se} \operatorname{cr}(G) \leq k$.

\footnotetext{
*Pesquisa financiada pela FAPESP Proc. 2014/14375-9, 2015/04385-0 e 2015/11937-9, CNPq Proc. 311373/2015-1
} 
Conhece-se na literatura um algoritmo ingênuo que dados $G$ e $k$, decide se $\operatorname{cr}(G) \leq k$. Este artigo apresenta um algoritmo que, apesar de não ser asintoticamente melhor, pode melhorar a complexidade em até $|E(G)|$ para grafos mais densos. Este artigo apresenta uma melhoria para algoritmo simples que determina se um grafo tem um ou menos cruzamentos. Na Seção 2 descreveremos o algoritmo ingênuo. Na Seção 3 descrevemos nosso algoritmo.

\section{Algoritmo Ingênuo para Decidir se $\operatorname{cr}(G) \leq k$}

Nesta seção descreveremos um algoritmo simples para decidir se $\operatorname{cr}(G) \leq k$.

Usamos indução em $k$. Se $k=0$ podemos usar um algoritmo de planaridade com complexidade linear no tamanho dos vértices [Hopcroft and Tarjan 1974].

Por indução em $k$, sabemos se $\operatorname{cr}(G)<k$. Se for, então o problema está resolvido. Suponha o contrário. Precisamos verificar se $\operatorname{cr}(G)=k$. Seja $e, f$ um par de arestas distintas de $G$. Denotamos por $G_{e, f}$ o grafo obtido subdividindo $e$ e $f$ e identificando as subdivisões. Denominamos este novo vértice de $v$. Note que se $D$ é um desenho de $G_{e, f}$ com $k-1$ cruzamentos $D$ também é um desenho de $G$ com $n+1$ cruzamentos, então, por hipótese, $G_{e, f}$ não pode ser desenhado com menos que $k-1$ cruzamentos.

Seja $e, f$ um par de arestas distintas de $G$. Por indução verificamos se $\operatorname{cr}\left(G_{e, f}\right)=$ $k-1$. Suponha que sim e seja $D$ um desenho de $G_{e, f}$ com $k-1$ cruzamentos. Logo, existe um desenho de $G$ com $k$ cruzamentos, e portanto $\operatorname{cr}(G)=k$. Se $n<k-1$, similarmente, temos que $\operatorname{cr}(G) \leq k-1$, contrariando nossa hipótese. Caso $\operatorname{cr}\left(G_{e, f}\right)>k-1$ temos que $G$ não possui nenhum desenho com no máximo $k$ cruzamentos tal que $e$ e $f$ se cruzam.

Logo se não existe nenhum par de arestas distintas $e, f$ de $G$ tal que $\operatorname{cr}\left(G_{e, f}\right) \leq$ $k-1$, concluímos que não existe nenhum desenho $D^{\prime}$ de $G \operatorname{com} \operatorname{cr}(D) \leq k$.

Note que a cada passo indutivo no algoritmo, adicionamos duas arestas e um vértice a mais no grafo. Portanto, são gerados no máximo $(|E(G)|+2 k)^{2}+1$ subproblemas e no caso base executamos um algoritmo de complexidade $O(|V(G)|+k)$. Logo, analisando a árvore da recursão, concluímos que o algoritmo tem complexidade $O\left((|E(G)|+2 k)^{2 k}(|V(G)|+k)\right)$.

\section{Reconhecendo Grafos com até um cruzamento}

Quando $k=1$ o algoritmo ingênuo descrito na seção anterior tem complexidade $O\left(|E(G)|^{2}|V(G)|\right)$. Nesta seção, descrevemos como podemos melhorar esse algoritmo neste caso.

Um par de arestas $e, f \in E(G)$ é um par cruzante se existe um desenho de $G$ com um cruzamento no qual $D[e]$ e $D[f]$ se cruzam. Um subgrafo $H$ de $G$ é um 1 -subgrafo de $G$ se $\operatorname{cr}(H)=1$. Seja $H$ um subgrafo de um grafo $G$. Denotamos por $D[H]$ o desenho de $H$ contido em $D$.

Lema 1. Seja $G$ um grafo não-planar. Se e, $f$ é um par cruzante de um grafo $G$ então e, $f$ também é um par cruzante de todo 1-subgrafo $H$ de $G$.

Demonstração. Seja $H$ um 1-subgrafo de $H$ e seja $D$ um desenho com um cruzamento de $G$ no qual e e $f$ se cruzam. Já que $D[H]$ tem que ter um cruzamento, o mesmo 
deve conter $e$ e $f$ pois são as únicas arestas que se cruzam em $D$. Logo, $D[H]$ tem um cruzamento.

O lema anterior mostra que não é necessário verificar se $\operatorname{cr}\left(G_{e, f}\right)=1$ para todo par de arestas de $G$. Basta apenas verificar para todo par de arestas cruzante de um 1subgrafo $H$ de $G$.

Assim, podemos modificar o algoritmo da seguinte maneira. Verificamos de $G$ é planar. Se sim então o problema está resolvido. Senão, existe um subgrafo $H$ de $G$ que é uma subdivisão de um $K_{3,3}$ ou $K_{5}$. O subgrafo $H$ pode ser obtido através do algoritmo de planaridade em tempo linear no número de vértices [Hopcroft and Tarjan 1974]. Verificamos se $\operatorname{cr}\left(G_{e, f}\right)=0$ para cada par de arestas $e, f$ distintas de $H$. Caso sim para algum par, então $\operatorname{cr}(G) \leq 1$. Caso contrário, $\operatorname{cr}(G)>1$.

$\mathrm{O}$ algoritmo tem complexidade $O\left(|E(H)|^{2}|V(G)|\right)$. No pior caso, no qual $G=$ $H$, a complexidade é a mesma do algoritmo descrito na seção anterior. Dado que o número de arestas de $H$ é linear com relação ao número de vértices de $G$, em grafos densos, temos uma melhora de até $|E(G)|$ no tempo de execução do algoritmo com relação ao ingênuo.

Podemos usar este algoritmo como caso base no algoritmo da seção anterior, melhorando o tempo de execução do mesmo. No caso em que é conhecido um 1-subgrafo $H$ de $G$ específico, podemos utilizar a informação sobre os pares cruzantes de $H$ para melhorar o tempo de execução.

\section{Referências}

[Garey and Johnson 1983] Garey, M. R. and Johnson, D. S. (1983). Crossing number is NP-complete. SIAM Journal on Algebraic Discrete Methods, 4(3):312-316.

[Grohe 2001] Grohe, M. (2001). Computing crossing numbers in quadratic time. In Proceedings of the Thirty-third Annual ACM Symposium on Theory of Computing, STOC '01, pages 231-236, New York, NY, USA. ACM.

[Hopcroft and Tarjan 1974] Hopcroft, J. and Tarjan, R. (1974). Efficient planarity testing. J. ACM, 21(4):549-568.

[Leighton 1983] Leighton, F. T. (1983). Complexity Issues in VLSI: Optimal Layouts for the Shuffle-exchange Graph and Other Networks. MIT Press, Cambridge, MA, USA.

[Purchase 1997] Purchase, H. (1997). Which aesthetic has the greatest effect on human understanding? In DiBattista, G., editor, Graph Drawing, volume 1353 of Lecture Notes in Computer Science, pages 248-261. Springer Berlin Heidelberg.

[Székely 1997] Székely, L. A. (1997). Crossing numbers and hard Erdős problems in discrete geometry. Combinatorics, Probability and Computing, 6:353-358. 